\title{
The effect of a dynamic increase in afterload on the first-phase ejection fraction
}

\author{
Katarzyna Cebrowska, Andrzej Minczykowski, Przemysław Guzik, Agnieszka Banaszak, Andrzej Wykrętowicz
}

Department of Cardiology Intensive Therapy, Poznan University of Medical Sciences, Poznań, Poland

Correspondence to: Andrzej Wykrętowicz MD, PhD,

Department of Cardiology Intensive Therapy, University School of Medicine,

Przybyszewskiego 49, 60-355 Poznań, Poland phone: +48618691391 e-mail: awykreto@ptkardio.pl Copyright by the Author(s), 2021

Kardiol Pol. 2021; 79 (7-8): 870-872; DOI: $10.33963 /$ KP.a2021.0041

Received: April 12, 2021 Revision accepted: June 17, 2021 Published online: June 17, 2021

\section{INTRODUCTION}

Ejection fraction (EF) is commonly accepted as a principal marker of left ventricular (LV) systolic function. This metric is helpful for the prediction of outcomes and to guide therapy in both moderately and severely impaired LV function. Chronic pressure overload in severe aortic stenosis (AS) leads to LV dysfunction. However, EF often remains normal in AS despite subclinical systolic dysfunction, thus, the subtle impairment of systolic function calls for a different diagnostic approach. Recently, Gu et al. [1] presented a first-phase ejection fraction (EF-1) as a novel, sensitive LV systolic function marker. The first-phase ejection fraction represents the percentage of LV volume change from the end-diastole to the peak of the aortic flow. It was demonstrated that reduced EF- 1 in subjects with AS and normal EF was associated with a poor prognosis and was considered a powerful predictor of adverse events in asymptomatic patients [2]. It was also shown that EF-1 in subjects with hypertension and normal EF was significantly diminished in those with concomitantly reduced diastolic function [1]. Thus, a chronic increase in afterload seems to contribute to the impairment of EF-1 during the phase of preserved total EF.

In the current investigation, we address whether, in healthy individuals, the acute increase in afterload affects total EF and firstphase ejection fraction similarly.

\section{METHODS}

Twenty healthy male volunteers were recruited. The participants were informed about the study, and their written consent was obtained. The local Ethics Committee approved the study protocol.

The study was performed as previously described [3]. In brief, echocardiographic tracings were acquired with a 3.5 MHz transducer (Vivid E95, GE Healthcare, Horten, Norway) at rest and at peak isometric handgrip (HG) - between 2 min $45 \mathrm{sec}$ and 3 min $15 \mathrm{sec}$ of maintaining $30 \%$ of maximal handgrip strength while lying in the left lateral recumbent position. Digital images were transferred to a computer workstation (EchoPAC, GE Healthcare, Horten, Norway) for offline analysis. Standard measurements of cardiac chambers' dimensions, volumes, and wall thickness were collected during diastole and systole according to the recommendations of the European Association and American Society of Echocardiography [4]. The LV end-diastolic volume (EDV), LV end-systolic volume, and LV ejection fraction were estimated as the mean value from four-chamber and two-chamber views according to the modified Simpson's rule [4]. A pulsed-wave Doppler (PW) was used to measure the peak velocity of the $E$ wave, whereas tissue Doppler echocardiography was used to quantify the peak early velocity of the $E^{\prime}$ wave at the level of the lateral and septal mitral annulus; these values were then used to calculate the average $\mathrm{E} / \mathrm{E}^{\prime}$ ratio.

\section{First-phase ejection fraction}

EF-1, a measure of early systolic ventricular function, is the percentage change in LV volume from end-diastole (EDV) to time of maximal velocity of aortic flow (T1V), and is calculated as follows:

$$
\mathrm{EF}-1=(\mathrm{EDV}-\mathrm{T} 1 \mathrm{~V}) / \mathrm{EDV} \times 100 \%[1]
$$

T1V as EDV was measured using the biplane modified Simpson's method.T1V was estimated using time difference measurements between the peak of the ECG R-wave to a maximal aortic flow velocity obtained from $\mathrm{PW}$ curves recorded in the LV outflow tract from an apical 5-chamber 
view. The PW gate was located close to the aortic valve leaflets level.

\section{Statistical analysis}

Normal distribution was evaluated with the D'Agostino-Pearson omnibus normality test. Continuous data are reported as the mean and standard deviation (SD). The differences between means were assessed using paired t-tests at a significance level of $P<0.05$. Relevant analyses were conducted using GraphPad Prism (Version 5, GraphPad Software, San Diego, CA, USA).

\section{RESULTS AND DISCUSSION}

The studied sample included 20 males at a mean age of 28.5 (3.9) years, with a weight of $79.6(10.3) \mathrm{kg}$, a height of 180.8 (6.9) cm, and LV mass index of $84.9(14.9) \mathrm{g} / \mathrm{m}^{2}$.

\section{Hemodynamic and echocardiographic response to isometric handgrip}

At the peak of the handgrip, systolic blood pressure, heart rate, and cardiac output were significantly elevated in comparison with values obtained at rest (Supplementary material, Table S1). Both stroke volume and E/E' remained unchanged at the peak of HG (Supplementary material, Table S1).

\section{Effect of afterload challenge on the first-phase ejection fraction and total ejection fraction}

The mean ejection fraction at resting state $(62.8 \%$ [6.3\%]) decreased significantly at the peak of handgrip (59.6\% [6.1]) (Figure 1A). In contrast, the mean EF-1 (20.2\% [5.4]) at rest increased significantly to $28.4 \%$ (7.1) (Figure 1B).

This study demonstrates a different effect of a rapid increase in LV afterload on ejection fraction and first-phase ejection fraction.

In healthy subjects, EF estimated at the peak of isometric handgrip was significantly diminished compared to those observed at rest. In contrast, a sudden increase in afterload was accompanied by a significant rise in EF-1.

Myocardial afterload during systole is determined by myocardial wall stress, which generates fiber shortening. Various mechanisms protect myocardial cells from excess load during blood ejection. In a typical "healthy" heart, lower myocardial wall stress occurs in the early phase of ejection. The novel marker, which allows assessing this phase in a clinical setting, is first-phase ejection fraction. Gu et al. [1] established that in hypertensive patients, EF-1 is impaired due to sustained myocardial contraction, which shifts myocardial wall stress into the late systole. Left ventricle afterload represents mechanical (hydraulic) load enforced by the arterial system on the left ventricle. During peak HG, the increase in blood pressure, systemic resistance, and arterial stiffness oppose blood ejection from LV. This resistance results in the diminished ejection fraction observed in our study. However, EF-1 at the peak of HG was significantly increased. This phenomenon

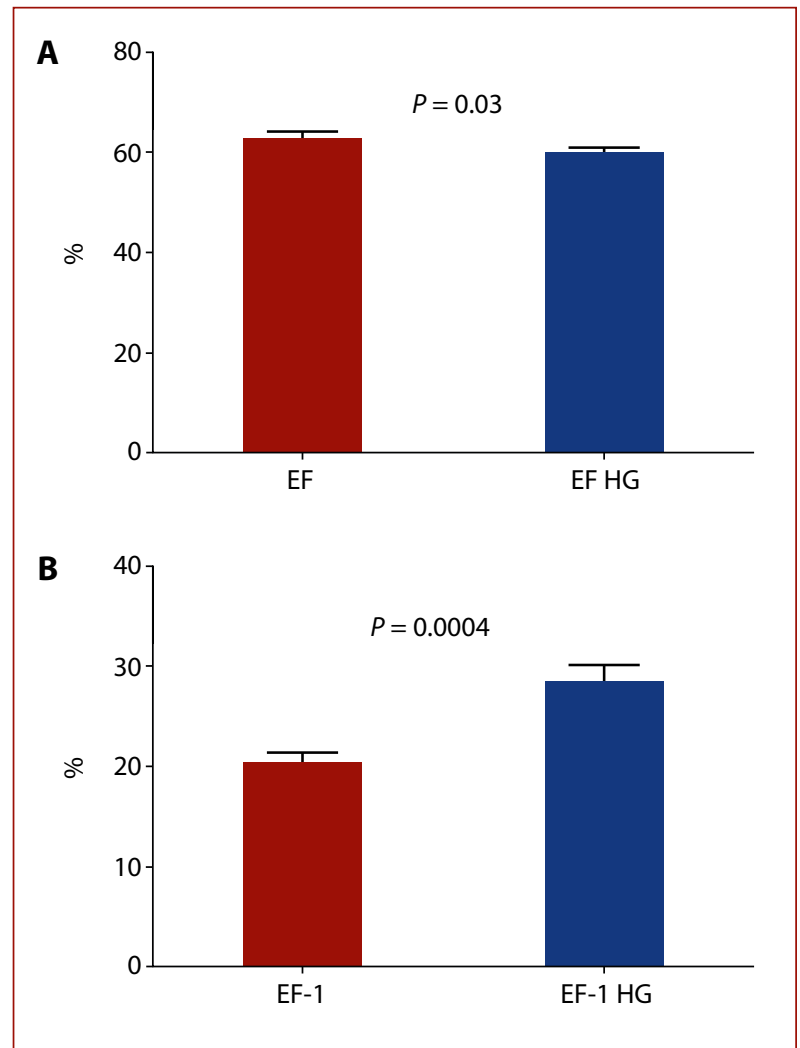

Figure 1 The effect of an acute increase in afterload during handgrip (HG) on ejection fraction (EF) and first-phase ejection fraction (EF-1). Data are presented as mean (SD)

may be explained by intrinsic heartbeat regulation. Reconditi et al. [5] demonstrated an integrated view of the Frank-Starling law combining mechanical, structural, and energetic aspects of heart performance during the systole-diastole cycle. They showed that mechanosensing in myosin filaments could adjust the number of myosin motors recruited to generate systolic force. In this mechanism, the energetic cost of a heartbeat is coordinated with the end-systolic pressure-volume relation. Therefore, it seems that the dysregulation of mechanosensing at the molecular level in various pathological conditions like hypertension leads to the loss of contractile efficiency and reduces EF-1. Along these lines, we demonstrated recently that at the peak of $\mathrm{HG}$, the reduction in ejection fraction in healthy subjects is accompanied by higher myocardial work and a better work efficiency index [3]. Moreover, Faconti et al. [6] showed a marked increase in EF-1 in recreational marathon runners after a race, despite the lack of increase in the conventional measure of systolic function.

\section{Study limitation and clinical relevance}

This study is regarded as a proof of concept; therefore, only healthy and young persons were included. Moreover, only one nonpharmacological mode of afterload challenge was used. Nevertheless, it seems that assessment of the dynamic challenge of the first-phase EF may be applied in 
various clinical settings like, e.g., heart failure, hypertension, or hypertrophic cardiomyopathy.

In summary, an acute increase in LV afterload results in a contrasting effect on the ejection fraction and EF-1 in healthy subjects.

\section{Supplementary material}

Supplementary material is available at https://journals. viamedica.pl/kardiologia_polska.

\section{Article information}

Conflict of interest: None declared.

Open access: This article is available in open access under Creative Common Attribution-Non-Commercial-No Derivatives 4.0 International (CC BY-NC-ND 4.0) license, allowing to download articles and share them with others as long as they credit the authors and the publisher, but without permission to change them in any way or use them commercially. For commercial use, please contact the journal office at kardiologiapolska@ptkardio.pl.

How to cite: Cebrowska K, Minczykowski A, Guzik P, et al. The effect of a dynamic increase in afterload on the first-phase ejection fraction. Kardiol Pol. 2021; 79(7-8): 870-872, doi: 10.33963/KP.a2021.0041.

\section{REFERENCES}

1. $\mathrm{GuH}, \mathrm{LiYe}$, FokH, et al. Reduced first-phase ejection fraction and sustained myocardial wall stress in hypertensive patients with diastolic dysfunction: a manifestation of impaired shortening deactivation that links systolic to diastolic dysfunction and preserves systolic ejection fraction. Hypertension. 2017; 69(4): 633-640, doi: 10.1161/HYPERTENSIONAHA.116.08545, indexed in Pubmed: 28223475.

2. Bing $\mathrm{R}, \mathrm{Gu} \mathrm{H}, \mathrm{Chin} \mathrm{C}$, et al. Determinants and prognostic value of echocardiographic first-phase ejection fraction in aortic stenosis. Heart. 2020; 106(16): 1236-1243, doi: 10.1136/heartjnl-2020-316684, indexed in Pubmed: 32345658.

3. Cebrowska K, Mińczykowski A, Krauze T, et al. The pressure-strain work indices in response to isometric handgrip exercise. Kardiol Pol. 2021; 79(4): 455-457, doi: 10.33963/KP.15912, indexed in Pubmed: 33784037.

4. Lang RM, Badano LP, Mor-Avi V, et al. Recommendations for cardiac chamber quantification by echocardiography in adults: an update from the American Society of Echocardiography and the European Association of Cardiovascular Imaging. J Am Soc Echocardiogr. 2015; 28(1): 1-39.e14, doi: 10.1016/j.echo.2014.10.003, indexed in Pubmed: 25559473.

5. Reconditi M, Caremani M, Pinzauti F, et al. Myosin filament activation in the heart is tuned to the mechanical task. Proc Natl Acad Sci U S A. 2017; 114(12): 3240-3245, doi: 10.1073/pnas.1619484114, indexed in Pubmed: 28265101.

6. Faconti L, Parsons I, Farukh B, et al. Post-exertional increase in first-phase ejection fraction in recreational marathon runners. JRSM CardiovasC Dis. 2020; 9: 2048004020926366, doi: 10.1177/2048004020926366, indexed in Pubmed: 32489656. 